
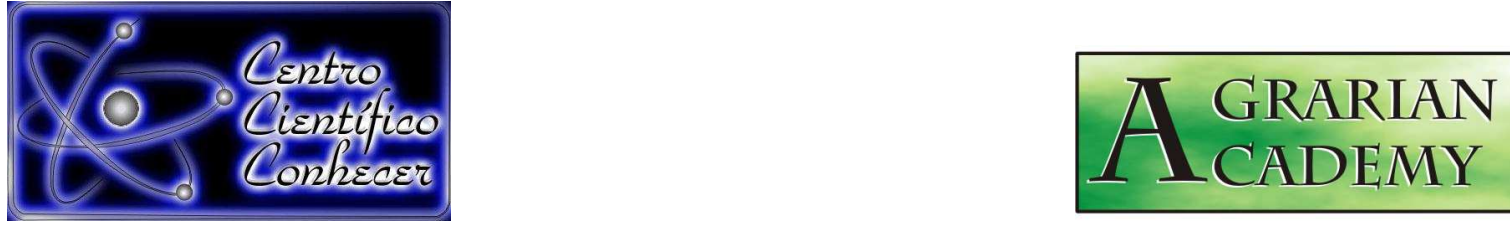

\title{
APLICAÇÃO DE SISTEMAS DE CRESCIMENTO E DA PRODUÇÃO DE Pinus taeda L. NO PLANALTO NORTE CATARINENSE
}

Ricardo Cavalheiro ${ }^{1}$, Sebastião do Amaral Machado ${ }^{2}$, Afonso Figueiredo Filho ${ }^{3}$, Allan Libanio Pelissari ${ }^{4}$

\footnotetext{
${ }^{1}$ Mestrando em Engenharia Florestal, UFPR - Universidade Federal do Paraná, Programa de Pós Graduação em Engenharia Florestal. Rua Lothário Meissner, 632 - Jardim Botânico 80210-170 - Curitiba, PR. E-mail: ricardocavalheiro11@gmail.com.

${ }^{2}$ Professor Sênior do Departamento de Ciências Florestais. UFPR - Universidade Federal do Paraná. Rua Lothário Meissner, 632 - Jardim Botânico 80210-170 - Curitiba, PR.

${ }^{3}$ Professor Associado do Departamento de Engenharia Florestal. UNICENTRO Universidade Estadual do Centro-Oeste PR-153 - KM 7 - Riozinho - 84500-000 - Irati, PR.

${ }^{4}$ Professor do Departamento de Ciências Florestais. UFPR - Universidade Federal do Paraná. Av. Pref. Lothário Meissner, 900 Jardim Botânico - 80210170 - Curitiba, PR.
}

Recebido em: 15/04/2017 - Aprovado em: 22/07/2017 - Publicado em: 31/07/2017 DOI: 10.18677/Agrarian_Academy_2017a6

\begin{abstract}
RESUMO
O objetivo deste estudo foi avaliar sistemas de crescimento e produção existentes na literatura florestal para estimativas do volume futuro em povoamentos de Pinus taeda L. Os dados utilizados neste trabalho foram provenientes de remedições de 104 parcelas permanentes, com formato quadrado de 0,04 ha de área. Foram avaliados quatro sistemas de modelos globais, quatro sistemas de modelagem em classes de diâmetros, um sistema para modelagem de árvores individuais e predições provenientes do SisPinus. $\mathrm{Na}$ avaliação, foram utilizadas as estatísticas de erro médio absoluto $\left(E_{a b s}\right)$ e relativo $\left(E_{r}\right)$, raiz quadrada do erro médio quadrático (REMQ) e índice de concordância de Willmott (d). Para avaliação da projeção do volume em diferentes classes de idade, as estatísticas de viés e acurácia foram empregadas. Na categoria de modelos globais a equação ajustada por Wolff II foi a mais precisa e acurada, assim como o sistema de Eisfeld para a modelagem em classes de diâmetro. Na modelagem por árvores individuais de Miranda, observaram-se resultados tendenciosos para a estimativa do crescimento em diâmetro e altura, apesar de resultados satisfatórios para a projeção do volume. $O$ simulador SisPinus subestimou o volume em todas as classes de idade. De acordo com os critérios estatísticos avaliados, dentre as categorias de modelos selecionadas, o sistema de equações global apresentado por Wolf II foi o mais preciso, acurado e não tendencioso para estimativa da produção em volume por unidade de área em diferentes classes de idade dos plantios avaliados.
\end{abstract}

PALAVRAS-CHAVE: Modelos Globais, Modelagem de Árvores Individuais, SisPinus. 


\title{
APPLICATION OF GROWTH AND YIELD PROGNOSIS SYSTEMS TO PinUS taeda L. PLANTATION IN THE NORTH OF SANTA CATARINA STATE
}

\begin{abstract}
The aim of this study was to evaluate available growth and yield systems in the forest literature for estimating the future volume of Pinus taeda $L$. stands. The data were obtained from 104 permanent plots, with square format of 0.04 ha of area. They were evaluated four Global Stand Models, four Diameter Distribution Models, one Individual Tree Model and estimates from SisPinus. In order to evaluate the projections, they were used the statistics of absolute (Eabs) and relative mean error (Er), square root of the quadratic mean square error (REMQ) and Willmott's concordance index (d). For evaluation the projection of the volume at different age classes, they were calculated the bias and accuracy statistics. In the category of Global Stand Models, the Wolff II equation presented higher statistics for volume estimation. The Eisfeld system was the most precise and accurate for the modeling by diameter classes. For the individual tree modeling the Miranda system presented biased results for estimation of diameter and height growth, despite satisfactory results for volume projection. The SisPinus simulator underestimated the volume at all age groups. According to the statistical criteria proposed, among the categories of selected models, the system of Global Stand Model presented by Wolf II was the most precise, accurate and non-biased to estimate volume yield at different age classes in the evaluated stands.
\end{abstract}

KEYWORDS: Global Stand Models, Diameter Distribution Models, Individual Tree Models, SisPinus.

\section{INTRODUÇÃO}

As decisões do manejo florestal a serem adotadas são baseadas em informações sobre o estoque presente e o crescimento futuro dos recursos existentes. Os inventários florestais fornecem as informações de volume somente no momento da medição. Contudo, as florestas são sistemas biológicos dinâmicos que estão em constante mudança (crescimento), em que se torna necessário projetar essas mudanças para obter informações relevantes na tomada de decisão (BURKHART \& TOMÉ, 2012).

Para isso, os modelos de crescimento e produção, são essenciais para guiar as estratégias do negócio florestal. Para o gênero Pinus no Brasil, a maioria das grandes empresas utilizam modelos generalizados de povoamentos totais (globais) para construção dos cenários de produção futura. Nesse gênero, o uso de técnicas mais complexas, como modelos de distribuição diamétrica e árvores individuais estudados por EISFELD et al., (2005), RETSLAFF (2014), VIANA (2016) e MIRANDA (2016) foram pouco avaliadas em grande escala e implementadas operacionalmente.

Grande parte dessa dificuldade se deve aos fatores: falta de séries temporais que abranjam todo o ciclo, ausência de dados consistentes, representatividade dos dados (sítios, idades e densidade), mudança do regime de manejo, troca de material genético e dificuldade na criação e implementação de scripts e softwares que utilizem um sistema de equações mais complexo.

Tendo em vista a importância do assunto no meio acadêmico e nas empresas florestais, torna-se imprescindível a condução de pesquisas que avaliem sistemas de modelagem da produção. Com isso o presente trabalho teve como objetivo avaliar sistemas de equações de crescimento e produção existentes na 
literatura florestal brasileira para povoamentos de Pinus taeda L., baseados na modelagem global, em classes diamétricas e árvores individuais. Também como complemento avaliou-se estimativas provenientes do simulador SisPinus.

\section{MATERIAL E MÉTODOS}

Os dados para a avaliação dos sistemas de crescimento e produção foram cedidos pela empresa WestRock localizada no município de Três Barras, Norte do estado de Santa Catarina. Foram provenientes de remedições de 104 parcelas permanentes alocadas em povoamentos não desbastados de Pinus taeda, com dimensões de $20 \mathrm{~m} \times 20 \mathrm{~m}$. As medições ocorreram ao longo de 24 anos a partir do ano de 1988, sendo que a amplitude de idade foi de cinco a 22 anos. As remedições das parcelas ocorreram nos intervalos de um ou dois anos resultando em 682 pares de dados remedidos. Nessa região, o clima é do tipo Mesotérmico Úmido (Cfb) segundo a classificação de Köppen. Para a estimativa do volume individual foi utilizada uma equação ajustada pela empresa WestRock a partir da cubagem de 360 árvores nas parcelas utilizadas neste estudo.

Para todos os sistemas avaliados a estimativa da altura dominante em função da idade, assim como a classificação de sítios (idade índice de 18 anos), foram realizadas em função da equação apresentada por MIRANDA (2016):

$$
h_{\text {dom }}=41,224399\left[1-e^{(-0,061832 l)}\right]^{1 / 156161}
$$

Em que: $h_{d o m}=$ altura dominante $(\mathrm{m})$; e $I=$ idade do povoamento (anos).

\section{Sistemas de modelos globais avaliados:}

Na Tabela 1 são apresentados por autor os sistemas de equações avaliados com suas respectivas estatísticas de ajuste e precisão. O primeiro sistema avaliado foi ajustado por WOLFF II (2012) para o modelo global de CLUTTER (1963). Os dados para realização do ajuste foram coletados a partir de análise de tronco completa de 144 árvores pertencentes a povoamentos de Pinus taeda localizados na região Centro Sul do estado do Paraná.

TABELA 1 - Modelos globais avaliados para projeção da produção nos povoamentos de Pinus taeda.

\begin{tabular}{|c|c|c|c|}
\hline Atributo & Equação & \multirow{2}{*}{$\begin{array}{l}R^{2} \\
\text { adj }\end{array}$} & \multirow{2}{*}{$\mathbf{S}_{Y X \%}$} \\
\hline & CLUTTER (1963) - WOLF II (2012) & & \\
\hline $\begin{array}{l}\text { Área } \\
\text { Basal } \\
\left(\mathrm{m}^{2} \cdot \mathrm{ha}^{-1}\right)\end{array}$ & $\operatorname{Ln}\left(G_{2}\right)=\operatorname{Ln}\left(G_{1}\right)\left(\frac{I_{1}}{I_{2}}\right)+4,8519\left(1-\frac{1}{I_{2}}\right)+0,0070\left(1-\frac{1}{I_{2}}\right) S+\varepsilon$ & 0,977 & 7,67 \\
\hline $\begin{array}{l}\text { Volume } \\
\left(\mathrm{m}^{3} \cdot \mathrm{ha}^{-1}\right)\end{array}$ & $\operatorname{Ln}\left(V_{2}\right)=3,3097+\left(\frac{-9,3418}{I_{2}}\right)+0,0134 S+0,7811 \operatorname{Ln}\left(G_{2}\right)+s$ & 0,977 & 10,66 \\
\hline \multicolumn{4}{|c|}{ CLUTTER (1963) - NASCIMENTO et al. (2015) } \\
\hline $\begin{array}{l}\text { Área } \\
\text { Basal } \\
\left(\mathrm{m}^{2} \cdot \mathrm{ha}^{-1}\right)\end{array}$ & $\operatorname{Ln}\left(G_{2}\right)=\operatorname{Ln}\left(G_{1}\right)\left(\frac{I_{1}}{I_{2}}\right)+4,6834\left(1-\frac{1}{I_{2}}\right)+0,0055\left(1-\frac{1}{I_{2}}\right) S+\varepsilon$ & 0,955 & 5,58 \\
\hline $\begin{array}{l}\text { Volume } \\
\left(\mathrm{m}^{3} \cdot \mathrm{ha}^{-1}\right)\end{array}$ & $\operatorname{Ln}\left(V_{2}\right)=2,9475+\left(\frac{-10,3349}{I_{2}}\right)+0,0068 S+0,9727 \operatorname{Ln}\left(G_{2}\right)+\varepsilon$ & 0,995 & 6,13 \\
\hline \multicolumn{4}{|c|}{ CLUTTER (1963) - ROMANIUK (2015) } \\
\hline Área & $\operatorname{Ln}\left(G_{2}\right)=\operatorname{Ln}\left(G_{1}\right)\left(\frac{l_{1}}{l_{2}}\right)+4,571\left(1-\frac{1}{l_{2}}\right)+0,021\left(1-\frac{1}{l_{2}}\right) S+\varepsilon$ & 0,650 & 9,05 \\
\hline
\end{tabular}

AGRARIAN ACADEMY, Centro Científico Conhecer - Goiânia, v.4, n.7; p. 65 


\begin{tabular}{cccc}
$\begin{array}{c}\left(\mathrm{m}^{2} \cdot \mathrm{ha}^{-1}\right) \\
\text { Volume } \\
\left(\mathrm{m}^{3} \cdot \mathrm{ha}^{-1}\right)\end{array}$ & $\operatorname{Ln}\left(\mathrm{V}_{2}\right)=2,453+\left(\frac{-4,564}{I_{2}}\right)+0,002 S+1,077 \operatorname{Ln}\left(G_{2}\right)+\varepsilon$ & 0,890 & 6,63 \\
\hline Área & SULLIVAN \& CLUTTER $(1972)-$ SCHUCHOVSKI (2014) & \\
$\begin{array}{c}\text { Basal } \\
\left(\mathrm{m}^{2} \cdot \mathrm{ha}^{-1}\right)\end{array}$ & $\ln \left(G_{2}\right)=\left(\frac{1}{I_{2}}-\frac{1}{I_{1}}\right) \ln \left(G_{1}\right)+3,6365\left[1-\left(\frac{I_{1}}{I_{2}}\right)\right]+0,05485\left[1-\left(\frac{I_{1}}{I_{2}}\right)\right]$ & 0,950 & 6,77 \\
$\begin{array}{c}\text { Volume } \\
\left(\mathrm{m}^{3} \cdot \mathrm{ha}^{-1}\right)\end{array}$ & $\ln \left(\mathrm{V}_{2}\right)=0,8425+0,03375-5,7676\left(\frac{1}{I_{2}}\right)+1,2406 \ln \left(G_{2}\right)$ & 0,974 & 8,66
\end{tabular}

Em que: $V_{2}=$ volume futuro $\left(\mathrm{m}^{3} \cdot \mathrm{ha}^{-1}\right) ; G_{2}=$ área basal estimada na idade de projeção $\left(\mathrm{m}^{2}\right.$.ha $\left.{ }^{-1}\right) ; G_{1}=$ número área basal na idade atual $\left(\mathrm{m}^{2}\right.$.ha $\left.{ }^{-1}\right) ; l_{2}=$ idade de projeção (anos); $l_{1}=$ idade inicial (anos); $N_{1}=$ número de árvores na idade atual (árv.ha ${ }^{-1}$ ); $S=$ índice de sítio $(\mathrm{m})$; e $\ln =$ logaritmo neperiano.

O segundo sistema de equações avaliado foi ajustado por NASCIMENTO et al., (2015) para o modelo global de CLUTTER (1963). Os dados utilizados na pesquisa foram provenientes da remedição de 216 parcelas permanentes instaladas em plantios desbastados de Pinus taeda localizados no norte do estado de Santa Catarina. O terceiro sistema foi ajustado por ROMANIUK (2015) que utilizou dados de 125 parcelas permanentes instaladas em plantios desbastados de Pinus taeda localizados no Centro Sul do estado do Paraná com área total de 1.000 ha.

O quarto sistema avaliado foi ajustado por SCHUCHOVSKI (2014) para o modelo global de SULLIVAN \& CLUTTER (1972). Essa autora utilizou dados de 1.472 parcelas permanentes instaladas em povoamentos desbastados de Pinus taeda localizados no Sudeste e Nordeste do estado do Paraná, Norte de Santa Catarina e Sudoeste de São Paulo.

\section{Sistemas de modelagem em classes de diâmetro avaliados:}

$\mathrm{Na}$ avaliação da modelagem em classes de diâmetro, optou-se por avaliar sistemas que utilizaram a FDP de Weibull com três parâmetros. Para a estimativa das alturas de cada classe de diâmetro em todas as avaliações foi utilizada a equação hipsométrica genérica ajustada por SCHUCHOVSKI (2014):

$$
h=5,19024+0,31069 d_{i}+1,35108 h_{d o m}-5,45576 \ln \left(h_{d o m}\right)-0,00624\left(d_{i} h_{d o m}\right)-0,09678 d_{g}
$$

Em que: $h=$ altura total estimada $(\mathrm{m}) ; h_{\text {dom }}=$ altura média das árvores dominantes na idade atual $(\mathrm{m}) ; d_{g}=$ diâmetro médio quadrático na idade de projeção; e $d_{i}=$ diâmetro do centro da classe $(\mathrm{cm})$.

$\mathrm{Na}$ ausência de uma equação ajustada, para as estimativas do diâmetro médio quadrático, variância dos diâmetros e da média aritmética do diâmetro à altura do peito foram utlizadas as seguintes formulações (SCOLFORO, 1990):

$$
d_{g}^{2}=\frac{G}{0,0000785398 N} \quad \bar{d}=\left(d_{g}^{2}-s^{2} d_{2}\right)^{0,5}
$$

Em que: $d_{g}=$ diâmetro médio quadrático; $d=\overline{\text { média aritmética dos diâmetros à }}$ altura do peito; $N=$ número de árvores estimado a partir do modelo de sobrevivência; $G$ = área basal estimada; e $s^{2} d_{2}=$ variância dos diâmetros. 
$\mathrm{Na}$ Tabela 2 são apresentadas as equações que compuseram os sistemas de projeção dos atributos do povoamento da modelagem em classes de diâmetro. $O$ primeiro sistema avaliado foi construído por VIANA (2016), que comparou dois métodos de obtenção dos parâmetros da FDP de Weibull na modelagem da produção de povoamentos de Pinus taeda localizados nos Campos Gerais do estado do Paraná. Foram utilizados dados de 826 parcelas permanentes. Optou-se nesse trabalho por avaliar esses dois métodos de obtenção dos parâmetros da FDP de Weibull. No primeiro método (Viana 1), fez-se a recuperação dos parâmetros via método dos momentos (SCOLFORO, 1998). No segundo método (Viana 2), os parâmetros da FDP de Weibull foram obtidos por predição com o uso das seguintes equações:

$$
\begin{gathered}
a=-0,9689+0,10141 d g+0,03284 d_{\max 2}+0,81809 d_{\min 2}+0,01632 I+0,00297 \frac{5 d_{2}}{\bar{d}}+0,01056 S \\
b=0,60169-1,134 a-0,062 d_{\max 2}+0,1250 d_{\min 2}+1,0789 d g \\
c=2,5689+0,46716 b+0,49076 d_{\min 2}-0,6253 d g+0,08641 \bar{d}
\end{gathered}
$$

Em que: $d g=$ diâmetro médio quadrático $(\mathrm{cm}) ; d_{\min 2}=$ diâmetro mínimo na idade de predição $(\mathrm{cm}) ; d_{\max 2}=$ diâmetro máximo na idade de predição $(\mathrm{cm}) ; I=$ Idade (anos); $s d_{2}=$ variância dos diâmetros na idade de predição; $\bar{d}=$ diâmetro médio aritmético (cm); $a$ = parâmetro de locação; $b=$ parâmetro de escala; e $c=$ parâmetro de forma.

\begin{tabular}{|c|c|c|c|}
\hline Atributo/Autor & Equação & $\begin{array}{l}R^{2} \\
\text { adj }\end{array}$ & $\mathbf{S}_{Y X \%}$ \\
\hline \multicolumn{4}{|c|}{ VIANA (2016) } \\
\hline $\begin{array}{c}\text { Sobrevivência } \\
{\left.\text { (árv.ha }{ }^{-1}\right)}^{\text {RETSLAFF }} \\
(2014)\end{array}$ & $N_{2}=N_{1}, \exp \left[-0,0204764\left(I_{2}-I_{1}\right)+0,0954373\left(\ln \left(\frac{I_{2}}{V_{1}}\right)\right)\right]$ & 0,976 & 2,10 \\
\hline $\begin{array}{l}\text { Área Basal } \\
\left(\mathrm{m}^{2} \cdot \text { ha }^{-1}\right) \\
\text { CLUTTER } \\
(1980)\end{array}$ & $G_{2}=0,021262+\ln \left(G_{1}\right)\left(\frac{T_{1}}{l_{2}}\right)+4,39364\left(1-\frac{l_{1}}{l_{2}}\right)+0,005066\left(1-\frac{l_{1}}{l_{2}}\right) S$ & 0,974 & 4,51 \\
\hline $\begin{array}{c}\text { Diâmetro } \\
\text { Mínimo (cm) } \\
\text { RETSLAFF } \\
(2014)\end{array}$ & $D_{\min 2}=0,6431+0,7629 D_{\min 1}-3,1226\left(\frac{I_{1}}{I_{2}}\right)+0,2451 d g_{2}$ & 0,829 & 15,50 \\
\hline $\begin{array}{c}\text { Variância dos } \\
\text { Diâmetros } \\
\text { SANTANA } \\
(2008) \\
\end{array}$ & $s^{2} d_{2}=0,1174+1,2271 s^{2} d_{1}-0,5510 G_{1}+0,4656 G_{2}$ & 0,937 & 21,03 \\
\hline $\begin{array}{c}\text { Diâmetro Máx. } \\
\text { NOGUEIRA } \\
\text { (2003) }\end{array}$ & 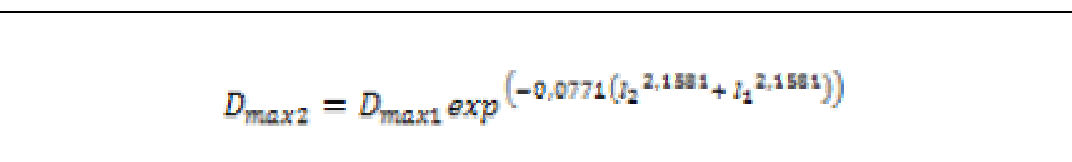 & 0,956 & 3,92 \\
\hline
\end{tabular}

TABELA 2 - Sistema de projeção dos atributos dos povoamentos de Pinus taeda ajustado por VIANA (2016). 
Sobrevivência

\begin{tabular}{|c|c|c|c|}
\hline $\begin{array}{l}\text { (árv.ha }^{-1} \text { ) } \\
\text { RETSLAFF } \\
(2014)\end{array}$ & 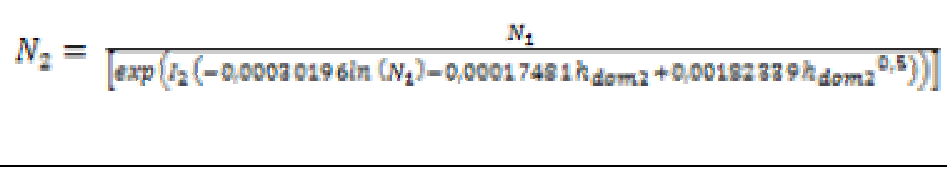 & 0,983 & 1,64 \\
\hline $\begin{array}{c}\text { Diâmetro } \\
\text { Médio } \\
\text { Quadrático } \\
\text { REINEKE } \\
\text { (1933) }\end{array}$ & $\log \left(N_{2}\right)=5,50923306-1,76714142 \log \left(\left(d g_{2}\right)\right.$ & 0,823 & 26,31 \\
\hline $\begin{array}{c}\text { Diâmetro } \\
\text { Médio } \\
\text { RETSLAFF } \\
(2014)\end{array}$ & $\overline{d_{2}}=-0,06293607+0.99024359\left(d g_{2}\right)$ & 0,992 & 0,60 \\
\hline \multicolumn{4}{|c|}{ EISFELD et al. (2005) } \\
\hline $\begin{array}{c}\text { Sobrevivência } \\
\text { (árv.ha }^{-1} \text { ) } \\
\text { SILVA \& } \\
\text { BAILEY (1987) }\end{array}$ & $N_{2} \equiv N_{1}, \exp \left[76,16092\left(0,999835^{l_{2}}=0,999835^{l_{1}}\right)\right]$ & 0,987 & 1,94 \\
\hline $\begin{array}{l}\text { Variância dos } \\
\text { Diâmetros } \\
\text { EISFELD et } \\
\text { al.,(2005) }\end{array}$ & $s^{2} d_{2}=1,0134 s^{2} d_{1}-0,1720 d g_{1}+4,7507\left(\frac{N_{1}}{N_{2}}\right)$ & 0,978 & 8,08 \\
\hline
\end{tabular}

Em que: $N_{2}=$ número de árvores na idade de projeção (árv.ha ${ }^{-1}$ ); $N_{1}=$ número de árvores na idade atual (árv.ha $\left.{ }^{-1}\right) ; l_{2}=$ idade de projeção (anos); $l_{1}=$ idade inicial (anos); $G_{2}$ = área basal estimada na idade de projeção $\left(\mathrm{m}^{2} \cdot \mathrm{ha}^{-1}\right) ; G_{1}=$ número área basal na idade atual $\left(\mathrm{m}^{2}\right.$.ha $\left.{ }^{-1}\right) ; S=$ índice de sítio; $h_{d o m 1}=$ altura média das árvores dominantes na idade atual $(\mathrm{m}) ; h_{d o m 2}=$ altura média das árvores dominantes na idade de predição $(\mathrm{m}) ; D_{\min 1}=$ diâmetro mínimo estimado na idade atual $(\mathrm{cm}) ; D_{\min 2}=$ diâmetro mínimo estimado na idade de projeção $(\mathrm{cm}) ; s^{2} d_{1}=$ variância dos diâmetros na idade atual; $s^{2} d_{2}=$ variância dos diâmetros na idade de projeção; $d g_{2}=$ diâmetro médio quadrático na idade de projeção; $D_{\max 1}=$ diâmetro máximo na idade atual $(\mathrm{cm}) ;$ e $D_{\max 2}=$ diâmetro máximo na idade futura $(\mathrm{cm}) ; d g_{1}=$ diâmetro médio quadrático na idade atual; e $d_{2}=$ média do diâmetro à altura do peito na idade futura (cm)

O segundo sistema de modelagem em classes de diâmetro avaliado foi composto por um sistema de equações ajustadas por RETSLAFF (2014) em estudo realizado com dados de povoamentos de Pinus taeda localizados em empresas privadas de várias regiões do estado do Paraná. A parcelas permanentes utilizadas nesse estudo totalizaram 1.023. Para esse sistema a obtenção dos parâmetros da FPD de Weibull foi realizada pelo método dos momentos (SCOLFORO, 1998).

O terceiro sistema avaliado foi construído por EISFELD et al., (2005) com dados de plantios de Pinus taeda localizados no município de Arapoti, estado do Paraná. A autora utilizou informações de 325 parcelas permanentes com duas a três medições. A obtenção dos parâmetros da FPD de Weibull também foi realizada pelo método dos momentos (SCOLFORO, 1998). 


\section{Sistema de modelagem de árvores individuais avaliado:}

O sistema de Árvores Individuais validado esta apresentado na Tabela 3. Esse conjunto de modelos foi ajustado por MIRANDA (2016) com dados de 1.396 parcelas permanentes de plantios de Pinus taeda localizados no município de Telêmaco Borba no estado do Paraná.

TABELA 3 - Sistema de árvores individuais de Pinus taeda ajustado por MIRANDA (2016).

\begin{tabular}{|c|c|c|}
\hline Atributo/Autor & Equação & $\mathbf{S}_{\mathbf{Y X} \%}$ \\
\hline $\begin{array}{c}\text { Prob. de } \\
\text { Mortalidade } \\
\text { MARTINS } \\
(2011)\end{array}$ & 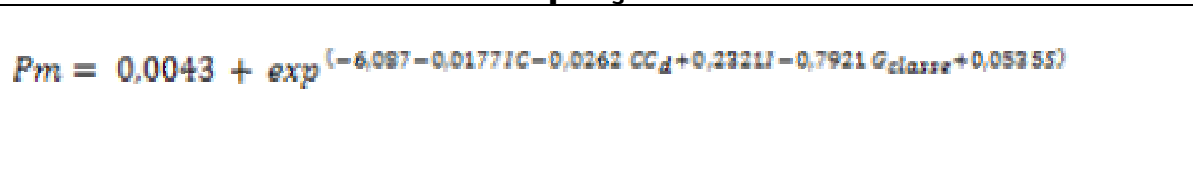 & 1,94 \\
\hline $\begin{array}{c}\text { Índice de } \\
\text { Competição } \\
\text { STAGE } \\
\text { (1973); } \\
\text { GLOVER \& } \\
\text { HOOL (1979). }\end{array}$ & $I C=\left(d_{i} \bar{d}^{-1}\right)^{z} ; I C_{1}=\left(d_{i} \bar{d}^{-1}\right)^{i} ; I C_{2}=\sum_{j=1}^{n} d_{j} d_{i}^{-1}$ & - \\
\hline $\begin{array}{c}\text { Cresc. em } \\
\text { Diâmetro }(\mathrm{cm}) \\
\text { MIRANDA } \\
(2016)\end{array}$ & $d_{2}=-1,0818+0,92299 d_{1}+0,12225 I_{2}+0,0967 S-0,0578 G+1,7812 I C_{1}$ & 5,76 \\
\hline $\begin{array}{c}\text { Crescimento } \\
\text { em Altura (m) } \\
\text { MIRANDA } \\
(2016)\end{array}$ & $h_{2}=1,1139+0,3231 h_{1}+0,6614 I_{2}+0,1468 S-0,0517 G-0,03322 I C_{2}$ & 5,91 \\
\hline
\end{tabular}

Em que: $P m=$ probabilidade de mortalidade por classe diamétrica; $I C_{i}=$ índice de competição; $C C_{d}=$ centro da classe diamétrica $(\mathrm{cm}) ; I=$ idade da parcela (anos); $G_{\text {classe }}=$ área basal da classe diamétrica $\left(\mathrm{m}^{2}\right.$.ha ${ }^{-1}$.classe); $d_{i}=$ diâmetro à altura do peito da árvore-objeto $(\mathrm{cm}) ; d_{j}=$ diâmetro à altura do peito das árvores competidoras $(\mathrm{cm}) ; d$ = média aritmética dos diâmetros à altura do peito das árvores da unidade amostral $(\mathrm{cm}) ; g_{i}=$ área transversal das árvores maiores à árvore-objeto $\left(\mathrm{m}^{2}\right) ; d_{2}=$ diâmetro à altura do peito na idade de projeção $(\mathrm{cm}) ; d_{1}=$ diâmetro à altura do peito na idade atual $(\mathrm{cm}) ; I_{2}=$ idade de projeção (anos); $S=$ índice de sítio; $G=$ área basal $\left(\mathrm{m}^{2}\right.$.ha $\left.{ }^{-1}\right) ; h_{2}=$ altura na idade de projeção $(\mathrm{m}) ;$ e $h_{1}=$ altura na idade atual $(\mathrm{m})$. Projeção do volume no SisPinus

Para a realização da projeção realizada no SisPinus, utilizou-se o FlorExel desenvolvido pela empresa Optimber. Os inputs das simulações foram: área basal $\left(\mathrm{m}^{2} \cdot \mathrm{ha}^{-1}\right)$, classificação de índice de sítio $(\mathrm{m})$ a partir da equação padrão do sistema, densidade (árv.ha ${ }^{-1}$ ) e a idade (anos), em que as condições eram conhecidas. $O$ grau de homogeneidade selecionado foi igual a 5 .

\section{Critérios estatísticos para avaliação dos sistemas validados:}

Para validar os sistemas de projeções testados, foram utilizadas as estatísticas de erros médios absoluto $\left(E_{a b s}\right)$, relativo $\left(E_{r}\right)$ e raíz quadrada do erro médio quadrático (REMQ). Também foi calculado o índice de concordância de WILLMOTT et al. (1985), que corresponde ao grau de associação entre as medidas 
projetadas e as reais, com variação de zero até um. Para facilitar a visualização dos desvios, foi realizada a análise gráfica de resíduos.

$$
\begin{aligned}
& E_{a b s}=\sum \frac{\left|y_{1}-\hat{y}_{1}\right|}{n} \quad E_{r}=E_{a b s} * \frac{100}{\bar{y}_{n}} \\
& R E M Q=\sqrt{\frac{\sum\left(y_{i}-\hat{y}_{i}\right)^{2}}{n}} \quad d=1-\left\{\frac{\sum\left(\hat{y}_{i}-y_{i}\right)^{2}}{\sum\left[\hat{y}_{i}-\bar{y}_{h}|+| y_{i}-\bar{y}_{h} \mid\right]^{2}+}\right\}
\end{aligned}
$$

Para a avaliação dos volumes projetados em classes de:

$$
\text { Viés }=\frac{1}{n} \sum_{i=1}^{n}\left(y_{i}-\widehat{y_{n}}\right) \text { Acurácia }=\frac{1}{n} \sum_{i=1}^{n}\left|y_{i}-\widehat{y}_{i}\right|
$$

Em que: $E_{a b s}=$ erro médio absoluto; $E_{r}=$ erro médio relativo; $R E M Q=$ raíz do quadrado médio; $y_{i}=$ valor observado; $y_{i}=$ valor estimado pela equação; $\bar{y}=$ valor médio da variável observada; $n=$ número de observações; $d=$ índice de concordância de Willmott; Viés = também conhecido por tendenciosidade ou "bias"; e Acurácia = mede em termos absolutos a proximidade de cada observação ao valor-alvo que se procura atingir.

Para avaliar a qualidade do ajuste da FDP de Weibull em relação as frequências observadas, foi aplicado o teste de aderência de Kolmogorov-Smirnov, ao nível de $5 \%$ de significância. As 104 parcelas avaliadas tiveram seus volumes observados confrontados com as projeções obtidas pelos sistemas sob avaliação. Para melhor visualização, os resultados foram divididos em quatro classes de idade. As projeções 7 a 10 anos de idade foram classificadas na classe de idade 1 , as parcelas com 11 a 14 anos foram classificadas na classe de 2, as parcelas com 15 a 18 anos foram classificadas na classe 3 e as parcelas com idades acima de 18 anos foram classificadas na classe 4.

\section{RESULTADOS E DISCUSSÃO}

\section{Projeções com modelos globais}

$\mathrm{Na}$ Tabela 4 pode-se observar as estatísticas de avaliação das equações utilizadas para as projeções do volume da idade inicial para todas as idades com remedição. A equação de WOLFF II (2012) apresentou os melhores resultados estatísticos, com $E_{a b s}=31,6 \mathrm{~m}^{3} \cdot \mathrm{ha}^{-1}, \mathrm{E}_{\mathrm{r}}=5,5 \%, \mathrm{REMQ}=41,8 \mathrm{~m}^{3}$.ha $\mathrm{h}^{-1}$ e $\mathrm{d}=0,983$.

TABELA 4 - Estatísticas de avaliação das equações utilizdas para modelagem global nos povoamentos de Pinus taeda.

\begin{tabular}{cccccc}
\hline Atributo & Autor & $\mathrm{E}_{\mathrm{abs}}$ & $\mathrm{E}_{\mathrm{r} \%}$ & $\mathrm{REMQ}$ & $\mathrm{d}$ \\
\hline \multirow{3}{*}{ Volume $\left(\mathrm{m}^{3} . \mathrm{ha}^{-1}\right)$} & WOLFF II (2012) & 31,6 & 5,5 & 41,8 & 0,983 \\
& NASCIMENTO et al., (2015) & 70,0 & 12,3 & 85,8 & 0,945 \\
& SCHUCHOVSKI (2014) & 271,5 & 47,8 & 327,1 & 0,632 \\
& ROMANIUK (2015) & 377,9 & 66,5 & 391,7 & 0,554 \\
\hline
\end{tabular}

Em que: $E_{a b s}=$ erro médio absoluto; $E_{r}=$ erro médio relativo; $R E M Q=$ raiz quadrada dos erros médios quadráticos; e $\mathrm{d}=$ índice de concordância de Willmott. 
$\mathrm{Na}$ Figura 1 estão apresentados os resíduos das projeções de volumes obtidas pelas quatro equações globais avaliadas. Observou-se que a equação de ROMANIUK (2015) apresentou tendenciosidade de superestimavas dos volumes em todas as parcelas, enquanto a equação de WOLF II (2012) foi a única que apresentou resíduos bem distribuídos em todas as idades, com apenas uma leve tendência de subestimativa nas idades acima de 20 anos, cujos desvios foram inferiores a $\pm 30 \%$ no geral.
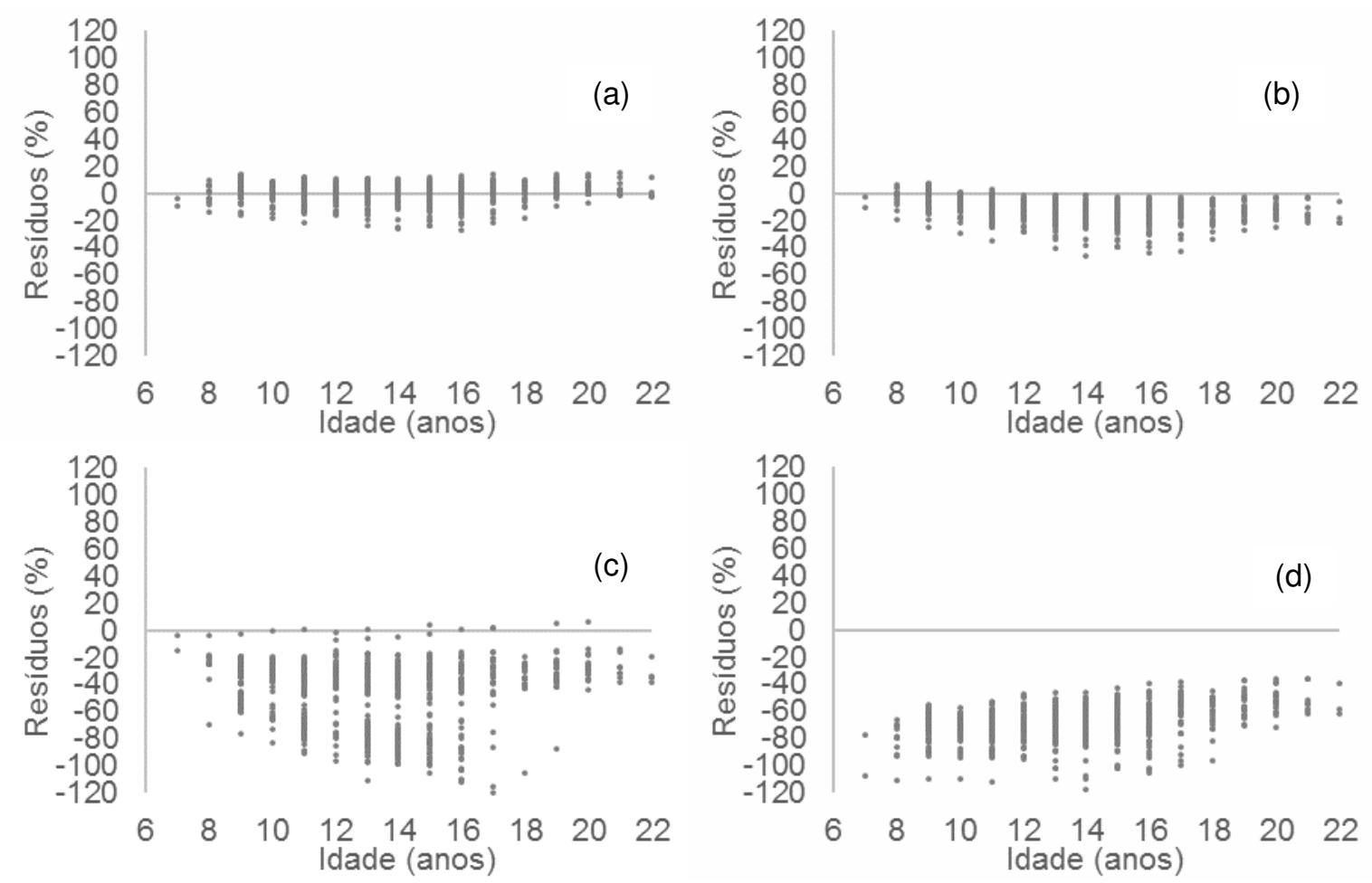

FIGURA 1 - Resíduos entre os volumes observados e os volumes projetados nos povoamentos de Pinus taeda pelas equações (a) WOLF II (2012); (b) NASCIMENTO (2015); (c) SCHUCHOVSKI (2014); E (d) ROMANIUK (2015).

As estatísticas da projeção do atributo volume em classes de idade são apresentadas na Tabela 5. No geral, a equação de WOLFF II (2012) apresentou o menor viés médio, com $13,6 \mathrm{~m}^{3} \cdot \mathrm{ha}^{-1}(1,7 \%)$. Na classe de idade 3 , de maior interresse devido a proximidade com a idade de rotação, a equação de WOLFF II (2012) também apresentou o melhor desempenho, com viés médio de $-0,7 \mathrm{~m}^{3}$.ha ${ }^{-1}$ ($0,7 \%$ ). A equação de ROMANIUK (2015) apresentou o pior viés médio, com -382,3 $\mathrm{m}^{3} \cdot \mathrm{ha}^{-1}(-65,5 \%)$.

Quanto à acurácia, o melhor desempenho também foi produzido pela equação ajustada por WOLF II (2012), onde os volumes estimados diferiram em média $34,6 \mathrm{~m}^{3}$.ha ${ }^{-1}(5,7 \%)$ dos volumes reais. As estimativas menos acuradas foram geradas pela equação de ROMANIUK (2015), com os volumes estimados diferindo em média $382,3 \mathrm{~m}^{3} \cdot$ ha $^{-1}(65,5 \%)$ dos reais. Nas projeções até 10 anos, a equação de NASCIMENTO et al., (2015) apresentou a melhor acurácia, com 17,9 m³. ha ${ }^{-1}$ $(5,5 \%)$.

Conforme os resultados apresentados, considerando os plantios em estudo, os piores resultados de projeção foram obtidos pelo sistema de ROMANIUK (2015). As possíveis causas dessa falta de precisão devem-se aos dados utilizados para 0 
ajuste das equações, uma vez que foram coletados em uma área relativamente pequena, com cerca de 1.000 ha, e em municípios com características diferentes dos plantios em estudo, não abrangendo assim todos os sítios. Além disso, os plantios foram desbastados em sua grande maioria com o objetivo final do uso da madeira para toras de grandes dimensões.

Por outro lado, o sistema de SCHUCHOVSKI (2014) teve dados de extensas áreas de plantio, no entanto, esses povoamentos estavam localizados em sítios, classes de solos e clima diferentes dos povoamentos avaliados nesta pesquisa. Os plantios compreendidos no trabalho dessa autora estavam localizados em grande maioria no Sudeste e Nordeste do estado do Paraná e Sudoeste do estado de São Paulo. Os plantios também apresentaram regimes de manejo com desbastes, cujo objetivo final de produção de madeira foi serraria e laminação.

TABELA 5 - Estatísticas de avaliação da projeção do volume por modelagem globral por unidade de área nos povoamentos de Pinus taeda.

\begin{tabular}{|c|c|c|c|c|c|c|}
\hline \multirow{2}{*}{ Atributo } & \multirow{2}{*}{ Autor } & \multicolumn{4}{|c|}{ Classes de Idade } & \multirow{2}{*}{ Média } \\
\hline & & 1 & 2 & 3 & 4 & \\
\hline \multirow{5}{*}{ 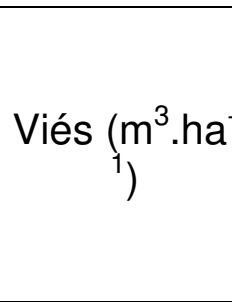 } & WOLFF II (2012) & 13,4 & 2,4 & $-0,7$ & 39,4 & 13,6 \\
\hline & $\begin{array}{l}\text { NASCIMENTO et } \\
\text { al., (2015) }\end{array}$ & $-12,2$ & $-65,5$ & $-105,8$ & $-109,6$ & $-73,2$ \\
\hline & SCHUCHOVSKI & & & & & \\
\hline & (2014) & $-139,1$ & $-287,4$ & $-352,0$ & $-244,7$ & $-255,8$ \\
\hline & ROMANIUK (2015) & $-246,0$ & $-377,4$ & $-459,3$ & $-446,6$ & $-382,3$ \\
\hline \multirow{4}{*}{$\begin{array}{l}\text { Acurácia } \\
\left(\mathrm{m}^{3} \cdot \mathrm{ha}^{-1}\right)\end{array}$} & WOLFF II (2012) & 21,1 & 27,1 & 42,6 & 47,5 & 34,6 \\
\hline & $\begin{array}{l}\text { NASCIMENTO et } \\
\text { al., (2015) } \\
\text { SCHIIHOYSI }\end{array}$ & 17,9 & 65,6 & 105,8 & 109,6 & 74,7 \\
\hline & $(2014)$ & 139,1 & 287,5 & 352,6 & 248,2 & 256,9 \\
\hline & ROMANIUK (2015) & 246,0 & 377,4 & 459,3 & 446,6 & 382,3 \\
\hline
\end{tabular}

A partir do uso das equações do trabalho de NASCIMENTO et al., (2015), os resultados das projeções do volume foram considerados satisfatórios. Os dados foram coletados em municípios e em regiões próximas aos plantios estudados, o que gerou condições similares de sítios, clima e solos. No entanto, esses plantios também tinham com objetivo final a obtenção de madeira de grandes dimensões, tendo recebido alguns desbastes. Estes também foram estabelecidos com material genético diferente do utilizado pela WestRock.

De acordo com todos os critérios estatísticos avaliados, o sistema de equações global apresentado por WOLF II (2012) foi considerado o mais preciso, acurado e não tendencioso para a estimativa da produção em volume em diferentes classes de idade nos plantios avaliados. Um dos principais motivos é que os locais onde os dados foram coletados por esse autor estão próximos de algumas fazendas da WestRock, fazendo com que essas áreas apresentam clima, solo e sítios muito semelhantes. Nessa região também é comum que as empresas façam o "benchmarking" dos tratos silviculturais, fazendo com que os plantios tenham padrões similares. Além disso, é de conhecimento que empresa adquiriu por muitos anos o material genético da WestRock, o que gerou padrão similar dos plantios e resultou em excelentes estimativas do volume futuro. 


\section{Prognoses com modelos em classes de diâmetro:}

As estatísticas do sistema de atributos construído por VIANA (2016) são apresentadas na Tabela 6. Em geral, somente as equações de estimativa do diâmetro mínimo e variância dos diâmetros apresentaram erros maiores que $10 \%$. Os atributos: números de árvores e diâmetro quadrático médio resultaram em índice de concordância maiores que 0,95 e erros menores que $4 \%$.

Nas estatísticas dos atributos pertencentes ao sistema de RETSLAFF (2014), o modelo de sobrevivência apresentou as melhores estatísticas, com $E_{a b s}=$ 73,6 árv.ha ${ }^{-1}, E_{r}=5 \%, R E M Q=119,7$ árv ha $^{-1}$ e $d=0,928$. O atributo variância dos diâmetros apresentou as estimativas mais imprecisas, com $E_{a b s}=10,2, E_{r}=48,6 \%$, $R E M Q=13,5$ e $d=0,436$. As estimativas da área basal também apresentaram-se imprecisas, com $E_{a b s}=13,3 \mathrm{~m}^{2} \cdot \mathrm{ha}^{-1}, \mathrm{E}_{\mathrm{r}}=20,8 \%, \mathrm{REMQ}=15,3$ e $\mathrm{d}=0,425$.

No sistema de EISFELD et al., (2005), somente as estimativas do atributo variância dos diâmetros apresentaram erros maiores que $10 \%$, no entanto, apresentaram alto índice de concordância $(0,928)$. A equação da estimativa da área basal apresentou boas estatísticas, com $\mathrm{E}_{\mathrm{abs}}=4,9 \mathrm{~m}^{2} \cdot \mathrm{ha}^{-1}, \mathrm{E}_{\mathrm{r}}=7,7 \%, \mathrm{REMQ}=6,2$ $\mathrm{m}^{2}$.ha ${ }^{-1}$ e $\mathrm{d}=0,907$. O método de estimativa do diâmetro médio apresentou os resultados mais imprecisos, com $E_{a b s}=2,4 \mathrm{~cm}, E_{r}=4,2 \%, R E M Q=3 \mathrm{~cm}$ e $d=$ 0,689 .

TABELA 6 - Estatísticas de avaliação das equações de atributos de VIANA (2016) utilizadas para modelagem em classes de diâmetro nos povoamentos de Pinus taeda.

\begin{tabular}{|c|c|c|c|c|}
\hline Atributo & $E_{\text {abs }}$ & $\mathrm{E}_{\mathrm{r} \%}$ & REMQ & $\mathrm{d}$ \\
\hline \multicolumn{5}{|c|}{ VIANA (2016) } \\
\hline Número de Árvores (árv.ha ${ }^{-1}$ ) & 54,2 & 3,7 & 79,0 & 0,969 \\
\hline Área Basal $\left(m^{2} \cdot\right.$ ha $\left.^{-1}\right)$ & 3,5 & 5,4 & 4,4 & 0,930 \\
\hline Diâmetro Quadrático $(\mathrm{cm})$ & 0,7 & 2,9 & 0,9 & 0,959 \\
\hline Diâmetro Médio (cm) & 1,3 & 6,0 & 1,8 & 0,813 \\
\hline Diâmetro Mínimo (cm) & 1,8 & 13,3 & 2,4 & 0,790 \\
\hline Variância dos Diâmetros & 3,5 & 16,7 & 4,8 & 0,927 \\
\hline Diâmetro Máximo (cm) & 1,7 & 5,2 & 2,5 & 0,893 \\
\hline \multicolumn{5}{|c|}{ RETSLAFF (2014) } \\
\hline Número de Árvores (árv.ha-1) & 73,6 & 5,0 & 119,7 & 0,928 \\
\hline Área Basal $\left(m^{2} \cdot h^{-1}\right)$ & 13,3 & 20,8 & 15,3 & 0,425 \\
\hline Diâmetro Quadrático $(\mathrm{cm})$ & 2,2 & 9,5 & 2,5 & 0,658 \\
\hline Diâmetro Mínimo (cm) & 2,5 & 18,7 & 3,0 & 0,487 \\
\hline Diâmetro Médio (cm) & 1,5 & 6,7 & 1,8 & 0,755 \\
\hline Variância dos Diâmetros & 10,2 & 48,6 & 13,5 & 0,436 \\
\hline \multicolumn{5}{|c|}{ EISFELD et al., (2005) } \\
\hline Número de Árvores (árv.ha ${ }^{-1}$ ) & 60,9 & 4,2 & 83,0 & 0,965 \\
\hline Área Basal $\left(\mathrm{m}^{2} . \mathrm{ha}^{-1}\right)$ & 4,9 & 7,7 & 6,2 & 0,907 \\
\hline Variância dos Diâmetros & 3,6 & 17,4 & 4,8 & 0,928 \\
\hline Diâmetro Médio $(\mathrm{cm})$ & 2,4 & 10,0 & 3,1 & 0,689 \\
\hline Diâmetro Quadrático $(\mathrm{cm})$ & 1,6 & 7,2 & 2,0 & 0,833 \\
\hline
\end{tabular}

Em que: $E_{a b s}=$ erro médio absoluto; $E_{r}=$ erro médio relativo; $R E M Q=$ raiz quadrada dos erros médios quadráticos; e $\mathrm{d}$ = índice de concordância de Willmott. 
$\mathrm{Na}$ Tabela 7 são apresentados os resultados do teste de aderência de Kolmogorov-Smirnov. Foi constatado que o sistema de EISFELD et al., (2005) apresentou a melhor aderência em relação às distribuições observadas, com $84 \%$ de aderência, KS médio de 0,124 e menor desvio padrão $(0,062)$. O sistema Viana 2 (2016) teve aderência de apenas $8 \%$ das parcelas, KS de 0,320 e desvio padrão de 0,159 .

TABELA 7 - Resumo dos resultados obtidos no teste de aderência de Kolmogorov-Smirnov

\begin{tabular}{|c|c|c|c|c|}
\hline \multirow[b]{2}{*}{ Autor } & \multirow[b]{2}{*}{ Dados } & \multirow{2}{*}{$\begin{array}{c}\text { Aderência } \\
\%\end{array}$} & \multicolumn{2}{|c|}{ Qualidade do Ajuste } \\
\hline & & & Média & $\begin{array}{l}\text { Desvio } \\
\text { padrão }\end{array}$ \\
\hline EISFELD & 682 & 84 & 0,124 & 0,062 \\
\hline 2016) & 68 & 7 & 0,129 & 0,061 \\
\hline RETSLAFF (2014) & 682 & 19 & 0,323 & 0,138 \\
\hline VIANA 2 (2016) & 682 & 8 & 0,320 & 0,159 \\
\hline
\end{tabular}

Em que: Não = total de parcelas que não aderiram ao teste de aderência; e $\mathrm{Sim}=$ total de parcelas que adereriram ao teste de aderência.

As estatísticas da projeção do atributo volume $\left(\mathrm{m}^{3} \cdot \mathrm{ha}^{-1}\right)$ são apresentadas na Tabela 8. O sistema avaliado de EISFELD et al., (2005) foi o que apresentou o menor viés médio, considerando todas as classes de idade, com superestimativa média de $21,8 \mathrm{~m}^{3}$.ha ${ }^{-1}$, equivalendo a $4,3 \%$. Esse sistema apresentou subestimativa média de 10,8\% dos volumes estimados nas idades abaixo de 10 anos e subestimativa dos volumes de $2,5 \%$ nas classes de idade acima de 18 anos.

Quanto a acurácia, o pior resultado foi apresentado pelo método de Viana 2 (2016), onde os volumes diferiram em média em $109,8 \mathrm{~m}^{3}$.ha $\mathrm{h}^{-1}(26,9 \%)$ dos reais. No entanto, esse sistema apresentou os resultados mais acurados nas projeções até 10 anos, gerando acurácia de $36,0 \mathrm{~m}^{3}$.ha ${ }^{-1}(11 \%)$.

Para a estimativa da produção futura em volume, EISFELD et al., (2005) obtiveram erro médio de $15,5 \mathrm{~m}^{3} \cdot \mathrm{ha}^{-1}$, sendo de $3,4 \%$. Para as idades acima de 18 anos, obtiveram erro médio de $11,1 \mathrm{~m}^{3}$. ha ${ }^{-1}$, equivalendo a $2,4 \%$.

TABELA 8 - Estatísticas finais da projeção do volume por unidade de área nos povoamentos de Pinus taeda.

\begin{tabular}{|c|c|c|c|c|c|c|}
\hline \multirow{2}{*}{ Atributo } & \multirow{2}{*}{ Autor } & \multicolumn{4}{|c|}{ Classes de Idade } & \multirow{2}{*}{ Média } \\
\hline & & 1 & 2 & 3 & 4 & \\
\hline \multirow{4}{*}{$\begin{array}{c}\text { Viés } \\
\left(\mathrm{m}^{3} \cdot \mathrm{ha}^{-1}\right)\end{array}$} & $\begin{array}{l}\text { EISFELD et al., } \\
(2005)\end{array}$ & 39,7 & 19,9 & 4,8 & 22,8 & \multirow{4}{*}{$\begin{array}{c}21,8 \\
90,7 \\
109,8 \\
192,8\end{array}$} \\
\hline & VIANA 1 (2016) & 51,0 & 80,6 & 104,3 & 127,1 & \\
\hline & VIANA 2 (2016) & $-10,3$ & 7,6 & 85,3 & 356,3 & \\
\hline & RESTSLAFF (2014) & 48,1 & 161,2 & 242,1 & 320,0 & \\
\hline \multirow{4}{*}{$\begin{array}{l}\text { Acurácia } \\
\left(\mathrm{m}^{3} \cdot \mathrm{ha}^{-1}\right)\end{array}$} & $\begin{array}{l}\text { EISFELD et al., } \\
(2005)\end{array}$ & 41,5 & 35,3 & 46,2 & 45,9 & \multirow{4}{*}{$\begin{array}{c}42,2 \\
91,2 \\
138,4 \\
197,5\end{array}$} \\
\hline & VIANA 1 (2016) & 51,1 & 81,3 & 105,5 & 127,1 & \\
\hline & VIANA 2 (2016) & 36,0 & 49,1 & 109,1 & 359,4 & \\
\hline & RETSLAFF (2014) & 65,9 & 162,0 & 242,1 & 320,0 & \\
\hline
\end{tabular}


Na Figura 2 são apresentados os resíduos das estimativas obtidas pelos quatro sistemas de projeção do volume em classes de diâmetro. Pode-se observar que o sitemas de equações de VIANA 2 (2016) apresentou tendenciosidade de superestimavas dos volumes nas idades acima de 16 anos. Já o sistema de EISFELD et al., (2005) apresentou resíduos bem distribuídos em todas as idades com erros em geral menores que $20 \%$. Os volumes obtidos por RETSLAFF (2014) apresentaram tendenciosidade de subestimativa em todas as idades, com resíduos de até $+50 \%$.
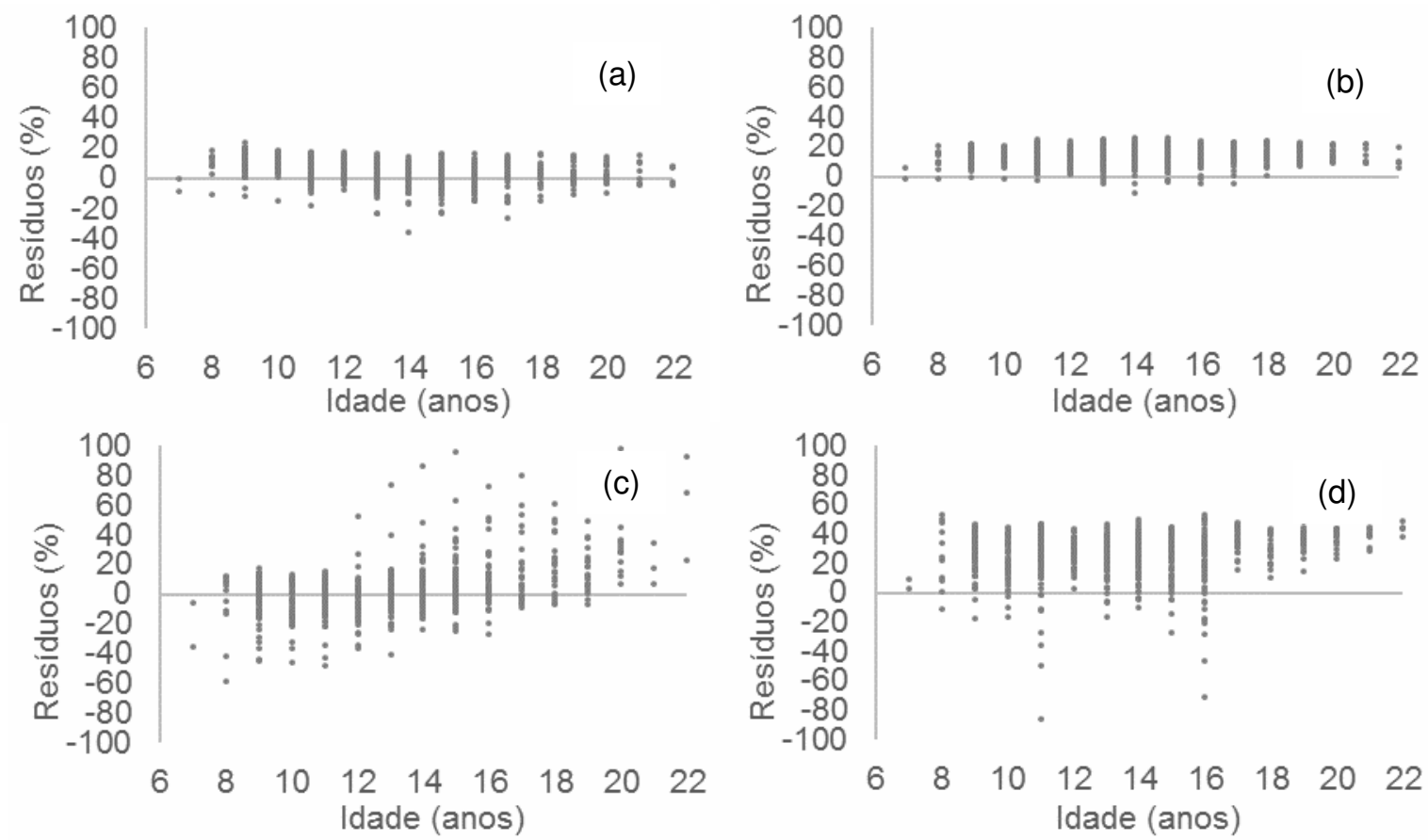

FIGURA 2 - Resíduos entre os volumes observados e os volumes projetados nos povoamentos de Pinus taeda pelos sistemas de equações. (a) EISFELD (2005); (b) VIANA 1 (2016); (c) VIANA 2 (2016); e (d) RETSLAFF (2014).

De forma geral o sistema de VIANA 1 (2016) apresentou resultados satisfatórios, mesmo com dados coletados em regiões distantes dos povoamentos estudados e com material genético diferente. O sistema de RETSLAFF (2014) apresentou estimativas imprecisas. Apesar de os plantios estarem localizados em regiões não tão distantes dos plantios avaliados observou-se que a principal causa dessas estimativas imprecisas foi o uso do Diagrama de Manejo da Densidade para estimativa do diâmetro quadrático médio comprometendo assim as estimativas da variância dos diâmetros e da área basal.

Para a modelagem do volume em classes de diâmetro o melhor sistema avaliado foi o de EISFELD et al., (2005). Essa precisão se deu ao fato de que o sistema de equações dos atributos se ajustou muito bem aos dados do estudo o que facilitou a obtenção dos parâmetros futuros da FDP de Weibull, gerando assim alta aderência das distribuições e estimativas de volume precisas e acuradas.

\section{Prognose com o modelo de árvores individuais:}

A Tabela 9 mostra as estatísticas de avaliação das equações utilizadas para estimativa dos atributos do povoamento pela modelagem de árvores individuias. A 
estimativa do incremento em altura apresentou os resultados mais imprecisos com $E_{a b s}=2,6 m, E_{r}=13,9 \%, R E M Q=3,3 \mathrm{me} \mathrm{d}=0,784$. Os resultados mais precisos, foram encontrados nas estimativas do número de árvores e incremento em diâmetro, com $E_{a b s}=39,4$ árv.ha ${ }^{-1}, E_{r}=2,7 \%, R E M Q=60,1$ árv.ha ${ }^{-1}$ e $d=0,982$ e $E_{a b s}=1,2$ $\mathrm{cm}, \mathrm{E}_{\mathrm{r}}=5,6 \%, \mathrm{REMQ}=1,7 \mathrm{~cm}$ e $\mathrm{d}=0,818$ respectivamente. As estimativas do volume também apresentaram excelentes resultados estatísticos com $E_{a b s}=49,8$ $\mathrm{m}^{3} \cdot \mathrm{ha}^{-1}, \mathrm{E}_{\mathrm{r}}=8,8 \%, \mathrm{REMQ}=63,4 \mathrm{~m}^{3} \cdot \mathrm{ha}^{-1}$ e $\mathrm{d}=0,962$.

TABELA 9 - Estatísticas de avaliação das equações utilizadas de MIRANDA (2016) para modelagem de árvores individuais nos povoamentos de Pinus taeda.

\begin{tabular}{ccccc}
\hline Atributo & $\mathrm{E}_{\mathrm{abs}}$ & $\mathrm{E}_{\mathrm{r} \%}$ & $\mathrm{REMQ}$ & $\mathrm{d}$ \\
\hline Incremento em Diâmetro $(\mathrm{cm})$ & 1,2 & 5,6 & 1,7 & 0,818 \\
\hline Incremento em Altura $(\mathrm{m})$ & 2,6 & 13,9 & 3,3 & 0,784 \\
\hline Número de Árvores $\left(\mathrm{m}^{3} \cdot \mathrm{ha}^{-1}\right)$ & 39,4 & 2,7 & 60,1 & 0,982 \\
\hline Área Basal $\left(\mathrm{m}^{2} \cdot \mathrm{ha}^{-1}\right)$ & 7,1 & 11,1 & 8,2 & 0,796 \\
\hline Volume $\left(\mathrm{m}^{3} \cdot \mathrm{ha}^{-1}\right)$ & 49,8 & 8,8 & 63,4 & 0,962
\end{tabular}

Em que: $E_{a b s}=$ erro médio absoluto; $E_{r}=$ erro médio relativo; $R E M Q$ = raiz quadrada dos erros médios quadráticos; $d$ = índice de concordância de Willmott.

\section{Comparação entre os sistemas de projeção testados:}

$\mathrm{Na}$ Tabela 10 são mostrados os resultados estatísticos das projeções do atributo volume obtidos por quatro diferentes métodos de simulação da produção. $\mathrm{Na}$ classe de idade 3, o volume foi superestimado em média $0,7 \mathrm{~m}^{3}$.ha' ${ }^{-1}$ pelo modelo global (MMG) de WOLF II (2012), bem como subestimado em 4,8 $\mathrm{m}^{3}$.ha ${ }^{-1}$ pela modelagem em classe de diâmetros (MCD) com o sistema de equações de EISFELD et al., (2005), e em 101,5 $\mathrm{m}^{3}$.ha ${ }^{-1}$ pelo SisPinus. Na classe de idade 4, o método de modelagem de árvores individuais (MAI) de MIRANDA (2016) teve o menor viés com 3,3 $\mathrm{m}^{3}$.ha ${ }^{-1}$. No geral, considerando todas as idades, o menor viés foi apresentado pelo MMG com 13,6 $\mathrm{m}^{3}$.ha ${ }^{-1}(1,7 \%)$.

Quanto a acurácia, os volumes obtidos pelo SisPinus diferiram em média 103,6 $\mathrm{m}^{3} \cdot$ ha $^{-1}(16,1 \%)$ dos volumes reais. Já a MCD apresentou acurácia média de $42,2 \mathrm{~m}^{3} \cdot$ ha $^{-1}(7,6 \%)$ e a MAl de $48,3 \mathrm{~m}^{3} \cdot \mathrm{ha}^{-1}(7,8 \%)$. O método mais acurado foi o de MMG, que apresentou acurácia de $34,6 \mathrm{~m}^{3}$.ha ${ }^{-1}(5,7 \%)$.

TABELA 10 - Estatísticas da projeção do volume em classes de idade por unidade de área nos povoamentos de Pinus taeda.

\begin{tabular}{|c|c|c|c|c|c|c|}
\hline \multirow{2}{*}{ Atributo } & \multirow{2}{*}{ Autor } & \multicolumn{5}{|c|}{ Classes de Idade } \\
\hline & & 1 & 2 & 3 & 4 & Média \\
\hline \multirow{4}{*}{$\begin{array}{l}\text { Viés } \\
\left(\mathrm{m}^{3} \cdot \mathrm{ha}^{-1}\right)\end{array}$} & $\begin{array}{c}\text { MMG (WOLFF II, } \\
\text { 2012) }\end{array}$ & 13,4 & 2,4 & $-0,7$ & 39,4 & 13,6 \\
\hline & $\begin{array}{c}\text { MCD (EISFELD et al., } \\
2005)\end{array}$ & 39,7 & 19,9 & 4,8 & 22,8 & 21,8 \\
\hline & MAI (MIRANDA, 2016) & 14,7 & 34,8 & 40,7 & 3,3 & 23,4 \\
\hline & SisPinus & 48,0 & 56,3 & 101,5 & 202,4 & 102,0 \\
\hline \multirow{4}{*}{$\begin{array}{l}\text { Acurácia } \\
\left(\mathrm{m}^{3} \cdot \mathrm{ha}^{-1}\right)\end{array}$} & $\begin{array}{l}\text { MMG (WOLFF II, } \\
\text { 2012) }\end{array}$ & 21,1 & 27,1 & 42,6 & 47,5 & 34,6 \\
\hline & $\begin{array}{c}\text { MCD (EISFELD et al., } \\
2005)\end{array}$ & 41,5 & 35,3 & 46,2 & 45,9 & 42,2 \\
\hline & MAI (MIRANDA, 2016) & 22,3 & 51,5 & 66,9 & 52,7 & 48,3 \\
\hline & SisPinus & 48,1 & 58,5 & 105,4 & 202,4 & 103,6 \\
\hline
\end{tabular}

AGRARIAN ACADEMY, Centro Científico Conhecer - Goiânia, v.4, n.7; p. 762017 
$\mathrm{Na}$ Figura 3 estão os resíduos das estimativas obtidas pelos quatro métodos de projeção do volume. No geral, MMG, MCD e MAI apresentaram distribuição de resíduos homogênoas em função da idade, com erros menores que $\pm 20 \%$. Observa-se que a MMG apresentou uma leve tendenciosidade de superestimativas dos volumes, enquanto o método de MCD apresentou sutil tendenciosidade de subestimativa dos volumes, com erros em geral inferiores a $20 \%$.
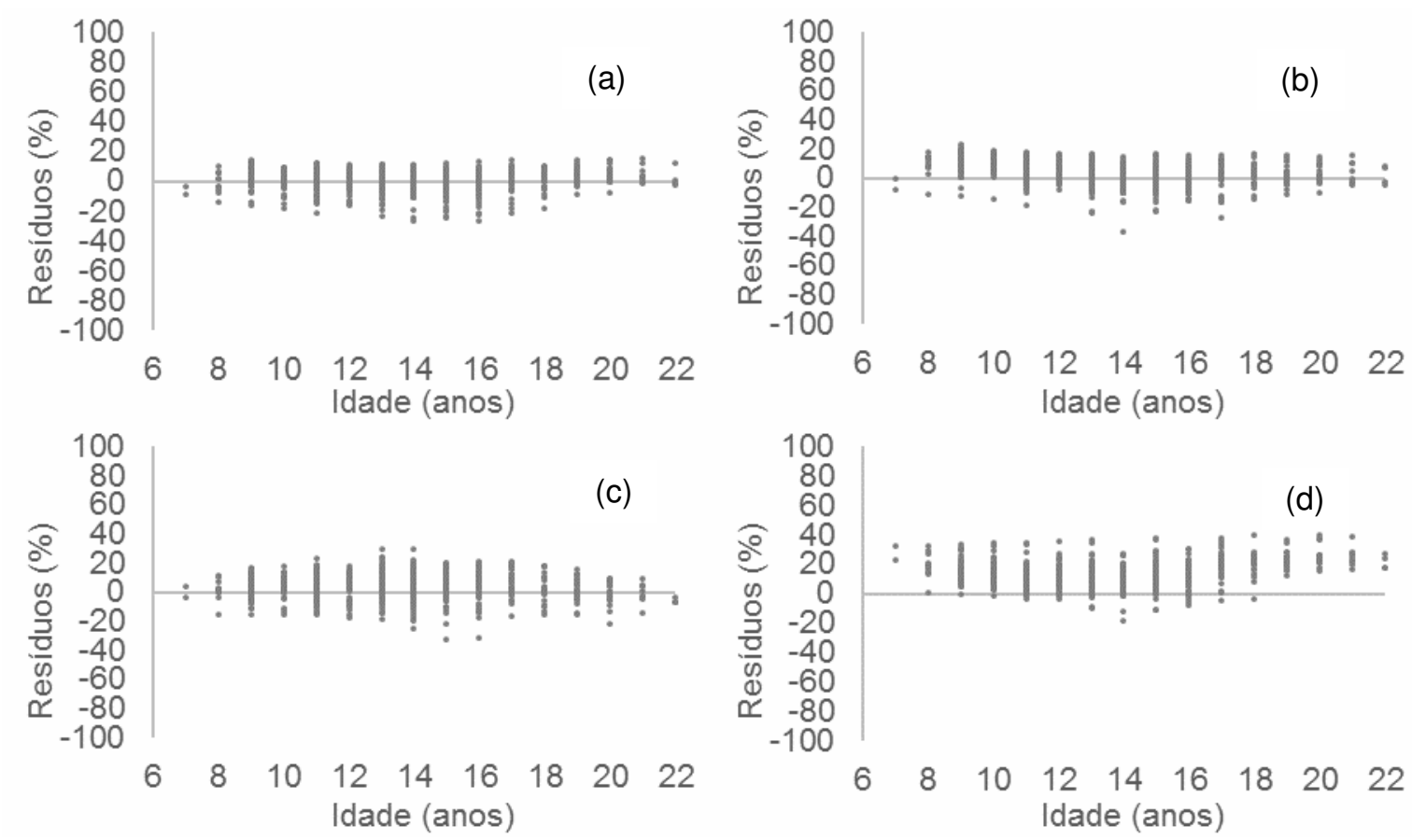

FIGURA 3 - Resíduos entre os volumes observados e os volumes projetados nos povoamentos de Pinus taeda pelos sistemas (a) WOLF II (2012); (b) NASCIMENTO (2015); (c) MIRANDA (2016); (d) SISPINUS.

\section{CONCLUSÕES}

Independente da categoria de modelos validados, observou-se relação entre a precisão da estimativa do volume nos povoamentos estudados e o sítio, o clima, os solos, o material genético e o regime de manejo onde as equações foram ajustadas.

$\mathrm{Na}$ categoria de equações globais as equações ajustadas por WOLF II (2012) são as mais precisas e acuradas para estimativa da produção em volume nos plantios avaliados. Para a modelagem em classes de diâmetro, o sistema de EISFELD et al., (2005) é o método mais preciso e acurado para a estimativa do volume.

Na modelagem por árvores individuais de MIRANDA (2016) observaram-se resultados tedenciosos para a estimativa do diâmetro, altura e área basal. No entanto, resultados satisfatórios são obtidos para a projeção do volume. Considerando todos os sistemas avaliados e os plantios do estudo, o sistema de modelagem global ajustado por WOLFF II (2012) é o sistema mais preciso, acurado e livre de tendências para a estimativa do volume em diferentes classes de idade.

\section{REFERÊNCIAS}

BURKHART, E. H.; TOMÉ, M. Modeling Forest Trees and Stands. New York: Springer, 2012. $460 \mathrm{p}$. 
CLUTTER, J. L. Compatible growth and yield models for loblolly pine. Forest Science, v. 9, n. 3, p. 354-371, 1963.

CLUTTER, J. L.; JONES, E. P. Prediction of growth after thinning in old field slash pine plantations. USDA. Forest Service. SE. Research Paper, 217: 1-14, 1980.

EISFELD, R. L; SANQUETTA, C.R; ARCE, J. E.; MAESTRI, R.; WEBER, K, S. Modelagem do crescimento e da produção de Pinus taeda L. por meio de função probabilística. Floresta, v. 35, n. 2, p. 317-328, 2005. Disponível em < http://dx.doi.org/10.5380/rf.v35i2.4619>. doi:10.5380/rf.v35i2.4619.

GLOVER, G. R.; HOOL, J. N. A basal area ratio predictor of loblolly pine plantation mortality. Forest Science, v. 25, n. 2, p. 275-282, 1979.

MARTINS, F. B. Modelagem de crescimento em nível de árvore individual para plantios comerciais de eucaliptos. Tese (Doutorado em Ciências Florestais) Universidade Federal de Viçosa, Viçosa, MG, 2011.

MIRANDA, R. O. V. Modelagem de árvores individuais para povoamentos não desbastados de Pinus taeda L. (Doutorado em Ciências Florestais) - Setor de Ciências Agrárias, Universidade Federal do Paraná, Curitiba, 2016.

NASCIMENTO, F. A. F.; DIAS, A. N.; FIGUEIREDO FILHO, A.; MIRANDA, G. M.; ARCE, J. Sistema de crescimento e produção para povoamentos de Pinus taeda na região norte de Santa Catarina. Cerne, v. 21, n. 2, p. 235-242, 2015. Disponível em $<\quad$ http://dx.doi.org/10.1590/01047760201521021494>. doi: 10.1590/01047760201521021494.

NOGUEIRA, G. S. Modelagem do crescimento e da produção de povoamentos de Eucalyptus sp e de Tectona grandis, submetidos a desbaste. Tese (Doutorado em Ciências Florestais) - Universidade Federal de Viçosa, Viçosa, MG, 2003.

REINEKE, L. H. Perfecting a stand-density index for even aged forests. Journal of Agricultural Research, v. 46, n. 7, p. 627-638, 1933.

RETSLAFF, F. A. de S. Simulador para prognose da produção de Pinus taeda com diagrama de manejo da densidade associado à modelagem em classes de diâmetro. Tese (Doutorado em Ciências Florestais) - Setor de Ciências Agrárias, Universidade Federal do Paraná, Curitiba, 2014.

ROMANIUK, D. S. Modelagem do crescimento e da produção em plantios de Pinus taeda L. na região Centro-Sul do Paraná. Dissertação (Mestrado em Ciências Florestais), Universidade Estadual do Centro Oeste, Irati, 2015.

SANTANA, C. J. de O. Simulação do crescimento e da produção em plantações desbastadas de Eucalyptus grandis com diferentes procedimentos de 
obtenção dos parâmetros da distribuição Weibull. Dissertação (Mestrado em Engenharia Florestal) - Universidade Federal do Paraná, Curitiba, 2008.

SCHUCHOVSKI, M. S. Modelos alométricos e de produção para plantações de Pinus taeda nos estados do Paraná e Santa Catarina. Tese (Doutorado em Ciências Florestais) - Setor de Ciências Agrárias, Universidade Federal do Paraná, Curitiba, 2014.

SCOLFORO, J. R. S. Sistema integrado para predição e análise presente e futura do crescimento e produção, com otimização de remuneração de capitais, para Pinus caribaea var. hondurensis. Tese (Doutorado em Ciências Florestais) - Setor de Ciências Agrárias, Universidade Federal do Paraná, Curitiba, 1990.

SCOLFORO, J. R. S. Modelagem do crescimento e da produção de florestas plantadas e nativas. Lavras: UFLA/FAEPE, 441 p., 1998.

SILVA, J. A. A.; BAILEY, R. A. Predicting diameter distribution in fertilized slash pine plantations with the Weibull distribution. Acta Forestalia Brasiliensis, n. 2, v. 1, p. 47-62, 1987.

STAGE, A. R. Prognosis model for stand development. USDA Forest Service: Research Paper INT-137, 1973. 32p.

SULLIVAN, A. D.; CLUTTER J. L. A simultaneous growth and yield model for loblolly pine. Forest Science, n. 18, p. 76-86, 1972.

VIANA, J. J. Modelagem do crescimento e da produção em classes de diâmetro para plantios de Pinus taeda L. não desbastados. Dissertação (Mestrado em Ciências Florestais), Universidade Estadual do Centro Oeste, Irati, 2016.

WILLMOTT, C. J.; ACKLESON, S. G.; DAVIS, R. E.; FEDDEMA, J. J.; KLINK, K. M.; LEGATES, D. R.; O'DONNELL, J.; ROWE, C. M. Statistics for the evaluation and comparison of models. Journal of Geophysical Research, v. 90, n. 5, p. 89959005, 1985.

WOLFF II, N. I. Modelagem do crescimento e produção de Pinus taeda L. Dissertação (Mestrado em Ciências Florestais), Universidade Estadual do Centro Oeste, Irati, 2012. 